\title{
JUVENTUD CHILENA: CULTURA DE MASA, MINORÍAS ACTIVAS Y TENSIONES DE ÉPOCA*
}

\author{
José Joaquín Brunner \\ Universidad Diego Portales
}

\begin{abstract}
RESUMEN: Este ensayo reflexiona sobre la cultura de los jóvenes en el Chile contemporáneo desde una triple perspectiva. Primero, desde el punto de vista de la cultura juvenil masiva, que, según muestran los datos de encuestas, expresa una conformidad subjetiva relativamente alta con sus condiciones actuales de vida. Segundo, desde el punto de vista de los grupos disidentes con el conformismo propio de la cultura juvenil de masa, que manifiestan una inserción conflictiva en la sociedad y revelan, más allá del consenso mayoritario, puntos de quiebre o zonas de integración social divergente. $\mathrm{Y}$ tercero, desde el punto de vista de una sociología crítica de la cultura, a partir de la cual se reflexiona sobre las circunstancias de época que deben enfrentar los jóvenes, especialmente en relación con procesos de racionalización, de mercantilización y de disolución de la autoridad paterna. Esta triple aproximación a la cultura de los jóvenes pretende poner en discusión el mito de la rebeldía etaria, subrayar las fuentes de disonancia normativa en la cultura juvenil y mostrar las dificultades de la integración social de los jóvenes en tiempos de modernidad tardía.

Palabras Clave: jóvenes, cultura juvenil, conformismo, minorías activas.
\end{abstract}

José JoAquín Brunner. PhD en sociología por la Universidad de Leiden, Países Bajos. Profesor titular de la Universidad Diego Portales. Miembro de número de la Academia de Ciencias Sociales, Políticas y Morales del Instituto de Chile. Email: josejoaquin.brunner@cpce.com.

* Versión revisada de la ponencia presentada a la Academia de Ciencias Sociales, Políticas y Morales el día 31 de julio de 2017. 


\title{
CHILEAN YOUTH: MASS CULTURE, ACTIVE MINORITIES AND TENSIONS OF OUR TIME
}

\begin{abstract}
This essay discusses contemporary Chilean youth culture from three different perspectives. First, from the point of view of massive juvenile culture as seen through survey data that show a relatively high degree of conformity with current life conditions. Secondly, form the point of view of groups that dissent form majority conformism expressed through massive youth culture and instead manifest a conflictive insertion in society and reveal, beyond the dominant consensus, breaking points and spaces of divergent social integration. And thirdly, from the point of view of cultural sociology, the circumstances of our time and situation that confront youth, both conformist majority and active minorities, are addressed, particularly in relation to processes of rationalization, commodification and dissolution of paternal authority. This threefold approach to youth culture seeks to call into discussion the myth of youth rebellion, to underline the sources of normative dissonance within juvenile culture, and to show the difficulties of young people's social integration in late modern times.
\end{abstract}

KEYWORDS: youth, youth culture, conformism, active minorities.

\section{MITO ROMÁNTICO Y AUTOPERCEPCIÓN ENCUESTADA}

66 enía veinte años. No dejaré que nadie diga que es la edad más bella de la vida". 'Así comienza un joven intelectual y novelista francés de izquierda, Paul Nizan, el libro de su viaje al Yemen en los años 30 del siglo pasado. Jean-Paul Sartre, compañero de curso del autor en la famosa L'École Normale Supérieure, más adelante dirá en su famoso prólogo a la reedición de esta novela en 1960: Nizan "puede (...) hablar de la juventud a nuestros jóvenes (...). Ellos reconocerán su propia voz. Y él puede decirles, a unos, os morís de modestia, atreveos a desear, sed insaciables, liberad las fuerzas terribles que dan vueltas bajo vuestra piel, no os avergoncéis de querer la luna: nos hace falta. Y,

${ }^{1}$ En el comienzo de su novela, Nizan escribe: "J'avais vingt ans. Je ne laisserai personne dire que c'est le plus bel âge de la vie. Tout menace de ruine un jeune homme: l'amour, la perte de sa famille, l'entrée parmi les grandes personnes. Il est dur à apprendre sa partie dans le monde". Paul Nizan, Aden, Arabie [1931], Ebooks libres et gratuits (2016), 4. Disponible en https://www.ebooksgratuits.com/pdf/nizan_aden_arabie.pdf. 
a los otros, dirigid vuestra rabia sobre los que la han provocado, no tratéis de escapar a vuestro mal, buscad sus causas y destruidlas". ${ }^{2}$

Este diálogo ilustra bien el mito de la juventud rebelde que, envuelta en los malestares de su sociedad y época, viviría indignada, llena de rabia, y soñaría con "cambiar la vida". Como proclamó nuestro Vicente Huidobro en su "Balance patriótico": "Entre la vieja y la nueva generación, la lucha va a empeñarse sin cuartel. Entre los hombres de ayer sin más ideales que el vientre y el bolsillo, y la juventud que se levanta pidiendo a gritos un Chile nuevo y grande, no hay tregua posible. Que los viejos se vayan a sus casas, no quieran que un día los jóvenes los echen al cementerio. (...) Que se vayan los viejos y que venga juventud limpia y fuerte, con los ojos iluminados de entusiasmo y de esperanza". 3

Este mito, dice la antropóloga Susanne Schröter, habla de una rebelión de los jóvenes dirigida no sólo contra las autoridades del Estado, sino contra la sociedad como un todo. Esto, agrega, torna a "las subculturas juveniles en algo único dentro del amplio espectro de las rebeliones; se rebelan frente a su propia sociedad, los padres, profesores y las generaciones más viejas en general, y además contra la mayoría de sus compañeros de edad. Consecuentemente, su conducta es un rechazo activo de las costumbres, valores y reglas vigentes". 4

A propósito del ciclo de protestas estudiantiles iniciado en 2006, también nosotros en Chile revivimos, y algunos exaltaron, este mito. Por momentos, incluso, los adultos rindieron pleitesía al mito de la excepcionalidad juvenil y renunciaron a su rol educativo, entregando a los jóvenes la responsabilidad de conducirlos. En esos momentos, venían a la memoria las palabras de Hannah Arendt a propósito de un asunto dis-

2 "Jeune et violent, frappé de mort violente, Nizan peut sortir du rang, parler de la jeunesse à nos jeunes gens (...) Ils reconnaîtront leur propre voix. Il peut dire aux uns: vous mourrez de modestie, osez désirer, soyez insatiables, délivrez les forces terribles qui se font la guerre et tournent en rond sous votre peau, ne rougissez pas de vouloir la lune: il nous la faut. Et aux autres: dirigez votre rage sur ceux qui l'ont provoquée, n'essayez pas d'échapper à votre mal, cherchez ses causes et cassez-les". Jean-Paul Sartre, "Préface”, en Aden, Arabie (París: Maspéro, 1976), 16.

${ }^{3}$ Vicente Huidobro, "Balance patriótico", Acción 4, 8 de agosto de 1925. Disponible en http://www.memoriachilena.cl/602/w3-article-317954.html.

${ }^{4}$ Susanne Schröter, "Rituals of Rebellion - Rebellion as Rituals: A Theory Reconsidered", en The Dynamics of Changing Rituals, editado por J. Kreinath, C. Hartung \& A. Deschner (Nueva York: Peter Lang, 2004). 
tinto, pero atinente al caso: "La escena — decía ella - me parecía una fantástica caricatura de la educación progresista la cual, al abolir la autoridad de los adultos, implícitamente niega la responsabilidad de ellos respecto del mundo al cual han traído a sus hijos, rehusando su deber de adentrarlos en él". 5

Mi propósito en lo que sigue es cuestionar el mito de la juventud rebelde, ofrecer una visión diferente de la subjetividad y percepciones de los jóvenes chilenos, reflexionar sobre las minorías activas y ciertas subculturas juveniles y, al final, retomar —en perspectiva contemporánea- el tema de Arendt sobre el mundo que legamos a nuestros hijos y a sus hijos.

Partiré describiendo quiénes y cuántos son los jóvenes considerados como categoría sociodemográfica; exentos, por tanto, de cualquier inscripción en una narrativa mitológica.

Si definimos a los jóvenes como el grupo etario entre 15 y 29 años, el 24 por ciento de la población pertenece a él; esto es, cerca de 4,3 millones de personas en 2015, año de referencia para todas las estadísticas que citaré a continuación. ${ }^{6}$ La mitad de estos jóvenes cursa estudios de educación básica, media o superior, siendo esta situación la más habitual en el tramo de edad de 15 a 19 años ( 82 por ciento) y menos generalizada en el tramo de edad de 25 a 29 años ( 23 por ciento).

Ser joven en el Chile actual es, por tanto y ante todo, una experiencia de escolarización. ${ }^{7}$ Cuando no estudian, los jóvenes trabajan. Así ocurre con el 73 por ciento y el 84 por ciento, respectivamente, de aquellos graduados de la enseñanza secundaria y terciaria. ${ }^{8} \mathrm{~A}$ su turno, los que no estudian ni trabajan (nini) dentro del grupo de edad de 20 a 24 años representan el 15 por ciento de los hombres y 27 por ciento de las mujeres jóvenes. ${ }^{9}$ Una mayoría, cercana a dos de cada tres (63,8 por ciento) declara pertenecer a la clase media (superior, media

\footnotetext{
${ }^{5}$ Hannah Arendt, "Reflections on Little Rock", Dissent 6 (1959): 50.
}

${ }^{6}$ Datos tomados de Injuv, VIII Encuesta Nacional de Jóvenes, 2015. En adelante los datos estadísticos citados, salvo que se indique algo distinto, pertenecen a esta encuesta.

${ }^{7}$ Sobre la complejidad de esta experiencia y las expectativas que crea, ver Manuel Canales et al., "Salir del cuarto. Expectativas juveniles en el Chile de hoy", Última Década 44 (2016): 73-108.

${ }^{8}$ OECD, Education at a Glance 2016 (París: 2016).

${ }^{9}$ Ibídem. 
o inferior). ${ }^{10}$ Con todo, en el grupo de edad de 18 a 29 años, el 11 por ciento de estos jóvenes se halla en situación de pobreza por ingresos y 22,7 por ciento en situación de pobreza multidimensional. Son cifras que revelan cuán a medio camino se encuentra todavía Chile en su expectativa de transformarse en un país desarrollado.

En la cultura juvenil, educación y trabajo se hallan unidos por un lazo vocacional-utilitario. Se estudia para trabajar y se trabaja para prosperar en la vida. ¿Qué condiciones serían las más importantes — según los jóvenes - para que les vaya bien en la vida? El 41 por ciento menciona ser constante y trabajar responsablemente; el 16 por ciento, tener una buena educación; el 15 por ciento, recibir apoyo de los padres y la familia, y el 14 por ciento, tener iniciativas y metas claras. Se trata pues de una visión que, si bien no coincide en sus fundamentos con aquella transmitida, según Max Weber, por la ética protestante y el espíritu del capitalismo, ${ }^{11}$ comparte sin embargo con ella una misma orientación práctico-racional y metódica de la vida, orientada hacia fines y basada en una cierta ascética personal del trabajo. Por el contrario, tener suerte, fe en Dios o buenos contactos es considerado importante para alcanzar el bienestar sólo por alrededor del 5 por ciento de los jóvenes.

Junto a la educación y el trabajo, los jóvenes destacan la importancia de la familia y los amigos. En ambos frentes, el 88 por ciento y el 87 por ciento, respectivamente, declara estar satisfecho o muy satisfecho. Un porcentaje similar piensa que en cinco años más estará mejor, expectativa que se mantiene invariable desde 2006. Quienes estiman que estarán peor son menos del 2 por ciento durante el mismo período.

Entonces, ¿qué dirían nuestros jóvenes sobre el dictum de Paul Nizan relativo a la infelicidad de los 20 años? La gran mayoría lo consideraría equivocado.

${ }^{10}$ Centro de Estudios en Juventud, "III Estudio sobre valores en jóvenes 2013”. Santiago: Universidad Católica Silva Henríquez. Citado en Mario Sandoval Manríquez, "Jóvenes y valores: Reflexiones sociológicas a partir de datos empíricos", en Juventudes: metáforas del Chile contemporáneo, editado por Pablo Cottet (Santiago: Proyecto Juventudes - RIL Editores, 2015), 253-278.

${ }^{11}$ Max Weber, La ética protestante y el espíritu del capitalismo, introducción y edición crítica de Francisco Gil Villegas (México D.F.: Fondo de Cultura Económica, 2012). 
Efectivamente, el 85 por ciento se reconoce feliz o muy feliz, el 12 por ciento ni lo uno ni lo otro, y únicamente el 3 por ciento se considera no muy feliz o nada feliz. Dicho en breve: hay un alto nivel de bienestar subjetivo entre los jóvenes chilenos. ${ }^{12}$ En cambio, su apreciación respecto del bienestar de los demás, o sea, de la sociedad en que viven, contrasta con esa percepción positiva. ${ }^{13}$ Por ejemplo, los mismos jóvenes que poseen una expectativa optimista de su futuro personal piensan mayoritariamente que el país estará peor $(36,6$ por ciento) o igual que ahora $(29,5$ por ciento) en cinco años más.

Existe, pues, una brecha entre sentimientos positivos respecto de la propia situación personal y sentimientos negativos respecto del bienestar colectivo. Estos últimos se asocian además con un bajo interés por la política, reducida satisfacción con la democracia y una falta de identificación política con corrientes ideológicas de izquierda, derecha y centro. Asimismo, revelan una desconfianza social generalizada, tanto hacia los otros como hacia las instituciones.

En suma, el mundo juvenil cotidiano, masivo y regular, no aparece descontento en lo subjetivo con su vida privada ni motivado a participar en la esfera colectiva. No arrastra una carga explosiva de rebeldía latente. Tampoco refleja un difuso malestar o una disposición de repudio hacia el orden establecido. Lo cual no significa que se encuentre sumido en un conformismo puramente adaptativo. Sin plegarse ante la infelicidad de Nizan, tampoco cultiva la percepción panglosiana de habitar el mejor de los mundos posibles.

Más bien, los jóvenes muestran niveles mezclados de crítica y resignación, de pesimismo y optimismo; impresiones de progreso, pero también de vivir rodeados de intolerancia y mercantilización. Por ejemplo, en contraste con el bienestar y la felicidad manifestados más arriba, entre dos tercios y la mitad de los jóvenes de 18 a 24 años piensa que "en Chile las cosas se hacen mal y no [están] mejorando";

12 Esto vale también para la población adulta. Véase Ricardo González, ¿Malestar en Chile? Informe Encuesta CEP 2016 (Santiago: Centro de Estudios Públicos, 2017). adulta.

${ }^{13}$ Ibídem. En efecto, también ocurre así en el promedio de la población 
que, "en general, los niveles de respeto y tolerancia son muy bajos" y que "es difícil que las personas hagan algo por los demás si no esperan recibir algo a cambio". 14

\section{MINORÍAS ACTIVAS}

Dicho todo esto, conviene volverse ahora hacia la juventud a partir de sus "minorías activas", dejando en el trasfondo su existencia como estrato social masivo. ${ }^{15}$ En efecto, mientras la mayoría ejerce usualmente su influencia desde arriba y tiende a reflejar y promover el statu $q u o$, las minorías comprometidas con una causa o una esperanza, con un rechazo o una fuga hacia adelante, con una contracultura o un nuevo patrón de convivencia suelen ser portadoras de cambio en la sociedad, pero lo manifiestan desde abajo, cuestionando el conformismo y los consensos establecidos. Los jóvenes activos como minorías de cambio llaman la atención y son considerados rebeldes, o desajustados, o inconformes, precisamente porque se apartan del sentir de la mayoría, cuestionan el sentido común y no se doblegan ante los mandatos y la influencia de la autoridad.

Entre estas minorías se cuentan, por ejemplo, grupos capaces de escenificar su extrema infelicidad, o consumidos por una rabia que les quema la piel, o movidos por el ideal de "cambiar la vida", o situados en las fronteras del saber y las artes, o que protestan en las calles contra el sistema capitalista y las leyes del Estado burgués. Estas minorías activas, que las encuestas no captan ni reflejan, alimentan el relato de la juventud como estrato rebelde, rabioso, creativo, innovador o desajustado. Los jóvenes invocados por Huidobro y Sartre forman parte de esas

${ }^{14}$ Centro de Estudios Públicos, Encuesta CEP abril-mayo de 2017, base de datos. Agradezco a Ricardo González el procesamiento especial de datos por edad.

15 Sobre la distinción entre juventud "ordinaria" y grupos culturales juveniles "distintivos", "espectaculares" o "desviados", véase Paul Hodkinson, "Youth Cultures and the Rest of Life: Subcultures, Post-Subcultures and Beyond", Journal of Youth Studies 19, n. 5 (2016): 629-645. Sobre influencia de las minorías, ver el clásico volumen de Serge Moscovici, Social Influence and Social Change (Londres: Academic Press, 1976). Para una discusión reciente, ver John M. Levine y R. Scott Tindale, "Social Influence in Groups", en APA Handbook of Personality and Social Psychology: Vol. 2, editado por M. Mikulincer \& P. R. Shaver (Group Processes: 2015): 3-34. 
minorías. De hecho, su alta exposición mediática y la frecuente espectacularidad que alcanzan algunos de sus miembros llevan a confundir a veces esas subculturas juveniles — "tribus urbanas", por ejemplo_- ${ }^{16}$ con la cultura juvenil de masas.

Pienso, por ejemplo, en aquellos grupos que expresan una inserción idiosincrásica en circuitos de consumo ${ }^{17}$ y que, a partir de allí, elaboran estilos de vida caracterizados por una estética especial -en la que la vestimenta y ciertos símbolos suelen jugar un papel importante- ${ }^{18}$ o bien por patrones típicos de consumo alimentario, o por una intensa adhesión a un ídolo como ocurre con los clubes de fans.

$\mathrm{O}$ bien, puede tratarse de movimientos generacionales emergentes en campos especializados, como el campo académico o el de la literatura y las artes. Son jóvenes que se levantan como contendientes frente a los incumbentes que dominan un campo, buscando desplazarlos de las posiciones de poder e influencia y de las redes que controlan el acceso a recursos y prestigio. Como proclama Huidobro, nuestro poeta: "Entre la vieja y la nueva generación, la lucha va a empeñarse sin cuartel".

Alternativamente, estas minorías activas pueden ser núcleos dirigentes de movimientos juveniles protestatarios. ${ }^{19}$ Históricamente han servido para renovar el cuadro político tradicional con nuevas ideas y organizaciones. ${ }^{20}$ Además, a través de los medios de comunicación pue-

${ }^{16}$ Este es un tópico ampliamente estudiado por la literatura sobre las subculturas juveniles. Para un enfoque reciente, ver Paul Hodkinson, "Beyond Spectacular Specifics in the Study of Youth (sub)Cultures", Journal of Youth Studies 15, n. ${ }^{\circ}$ 5 (2012): 557-72. Para el caso de Chile, ver Óscar Aguilera Ruiz, "Los estudios sobre juventud en Chile: coordenadas para un estado del arte", Última Década 17, n. ${ }^{\circ} 31$ (2009): 109-127. Disponible en https://dx.doi.org/10.4067/S071822362009000200007.

17 Ver, por ejemplo, David Buckingham, "Selling Youth: The Paradoxical Empowerment of the Young Consumer", cap. 20. en Youth Cultures in the Age of Global Media, de D. Buckingham et al. (Basingstoke: Palgrave Macmillan, 2014).

18 Ver, por ejemplo, Paul Hodkinson, "Spectacular Youth? Young People's Fashion and Style", en Handbook of Youth and Young Adulthood, editado por A. Furlong (Londres: Routledge, 2009).

${ }^{19}$ Ver Rodrigo Miguel Medel Sierralta y Nicolás Manuel Somma González, "¿Marchas, ocupaciones o barricadas? Explorando los determinantes de las tácticas de la protesta en Chile", Política y Gobierno 23, n. ${ }^{\circ} 1$ (2016): 163-199.

${ }^{20}$ Ver, por ejemplo, Jennifer Earl et al., "Youth, Activism, and Social Movements", Sociology Compass 11, n. ${ }^{\circ} 4$ (2017), doi: 10.1111/soc4.12465. 
den incidir sobre la agenda de asuntos nacionales. El uso de las redes sociales incrementa su capacidad de automovilizarse. ${ }^{21}$

O pueden ser grupos, pandillas o colectivos surgidos de situaciones de desintegración social y anomia, como expresiones de rechazo al ordenamiento legal, de confrontación con los poderes dominantes, o de denuncia a la cultura y valores establecidos ${ }^{22}$. Estas conductas, magnificadas a veces por los media, llevan a reacciones de "pánico moral"23 frente a la cultura juvenil, retratada entonces como violenta, anárquica y disolvente del orden tradicional de la sociedad.

En suma, los motivos del activismo de las minorías juveniles pueden ser variados y aparecer en las más diversas áreas de actividad y de la vida pública o privada. Pueden estar vinculados a diferentes órdenes de la existencia social, desde la educación al trabajo, del consumo a las religiones, de la política al sexo, de la esfera de derechos a la esfera de las conductas desviadas.

Una reflexión sobre la cultura juvenil no puede limitarse únicamente a sus aspectos masivos, de opinión pública encuestada y satisfacción con los patrones establecidos y los valores dominantes. Si ésta fuese la única perspectiva de análisis empleada, sería imposible entender y dar cuenta del cambio, de la disconformidad, de la divergencia y anomia que pueden existir - y de hecho existen - al interior de esa cultura conformista, adaptativa, precisamente como contraculturas, disidencias, heterodoxias o propuestas alternativas, por marginales que sean.

${ }^{21}$ Cristián Cabalin, "Estudiantes conectados y movilizados: El uso de Facebook en las protestas estudiantiles en Chile", Comunicar, Revista Cientifica de Educomunicación 43, $\mathrm{n}^{\circ}$. XXII (2014): 25-33.

${ }^{22}$ Mariane Krause et al., "Social Representations of Violence among Young Chileans Involved in Violence", Psicoperspectivas, Individuo y Sociedad 13, n. ${ }^{\circ} 2$ (2014): 55-66.

23 "A condition, episode, person or group of persons emerges to become defined as a threat to societal values and interests; its nature is presented in a stylized and stereotypical fashion by the mass media; the moral barricades are manned by editors, bishops, politicians and other right-thinking people; socially accredited experts pronounce their diagnoses and solutions; ways of coping are evolved or (more often) resorted to; the condition then disappears, submerges or deteriorates and becomes more visible. (...) Sometimes the panic passes over and is forgotten (...); at other times it has more serious and long-lasting repercussions and might produce such changes as those in legal and social policy or even in the way society conceives itself'. Stanley Cohen, Folk Devils and Moral Panics (Londres: MacGibon and Kee, 1972), 9. 


\section{HERENCIA CULTURAL}

¿Cuánto contribuyen la propia sociedad y la cultura establecida a moldear a esas minorías activas y a condicionar sus subculturas? Esta pregunta es clave, pues puede servir como un indicador de futuras evoluciones de las culturas juveniles. En efecto, las minorías se activan no sólo en términos de sus propias motivaciones de rechazo o disconformidad o rebeldía frente al orden establecido. Lo hacen, además, en función de las condiciones de la sociedad en que ellas nacen y que no eligen, las cuales sin embargo determinan los parámetros esenciales de su tiempo y espacio, de la cultura de su época, del ethos de la sociedad en que están llamadas a desenvolverse y en que deben transformarse de niños en adultos.

No es posible, claro está, dentro de los apretados límites de este ensayo, hacer un diagnóstico de la época, un recuento del zeitgeist en que a los jóvenes actuales les toca hacer su vida y crear una identidad cultural. Sin embargo, es necesario. De lo contrario, nos quedaríamos sin una perspectiva externa a la cultura de los propios jóvenes como estrato masivo, y de sus minorías activas, que nos permita evaluar el momento histórico en que deben crecer y formarse para luego hacerse cargo gradualmente de la conducción de su sociedad.

Aquí se abre, pues, un amplio espacio de indagación sobre la cultura contemporánea y su probable impacto sobre la juventud, tanto en sus circuitos de masas como de minorías activas, pero especialmente estas últimas, ya que esas minorías juveniles son las que más intensamente viven el drama de su tiempo. Y éste puede llevarlas a pensar que se hallan en "la edad más bella de la vida" o en la más aborrecible, o en cualquier situación intermedia.

Para poder abordar de una manera ultra sucinta tan graves y complejos asuntos, me limito a mencionar tres tipos o familias de fenómenos de la cultura contemporánea que, creo, revisten especial importancia para la evolución de las culturas juveniles.

Primero, el fenómeno weberiano de la racionalización instrumental y el desencantamiento del mundo. ${ }^{24}$ Es bien sabido que Weber

${ }^{24}$ Lawrence A. Scaff, "Max Weber's Legacy”, presentación en la reunión anual de la Kantoh Sociological Society, Tokyo, 22 de junio de 2014. Disponible en: https://www.jstage.jst.go.jp/article/kantoh/2015/28/2015_18/_pdf. 
otorgaba un lugar central en su interpretación de la cultura de Occidente a estos procesos que acompañan al desenvolvimiento de las burocracias y los mercados y a la cientifización y tecnificación del mundo. Creaban, decía él, una "jaula de hierro" en que la experiencia humana queda atrapada y se constriñe cada vez más hasta tornarse mera administración técnica e intercambio utilitario, ahogando "las únicas cuestiones que nos importan: las de qué debemos hacer y cómo debemos vivir". ${ }^{25}$

Pregunta: ¿acaso no hay entre los jóvenes — de manera explosiva a veces, a veces como un sordo malestar o mediante el retraimiento de la polis - un deseo cada vez mayor por escapar de esa jaula y salir al encuentro de un mundo con múltiples racionalidades, afectiva, mística, de valores plurales, de compromiso y testimonio, de reciprocidad o de reconciliación con la naturaleza? ¿No es éste, acaso, el motivo de numerosas y diversas reacciones contra la mecanización del mundo, las mediciones constantes, el predominio abstracto de las organizaciones y la reducción de los procesos vitales a un cada vez más minucioso juego de causas y efectos, pero no necesariamente de sentidos y sinsentidos?

Segundo, el fenómeno marxiano de la mercantilización; esto es, la conversión del trabajo, el conocimiento, la educación, el arte, el tiempo, la naturaleza y la vida erótica en mercancías, en coyunturas de intercambio, en oferta y demanda de bienes que, expresados en dinero, circulan por las redes de la economía, la sociedad, la política y la cultura. Igual que Weber, Marx reconocía el enorme poder de los mercados y su capacidad infinitamente invasiva, capaz de "colonizar" todos los

${ }^{25}$ Max Weber, en su ensayo "La ciencia como vocación”, plantea la cuestión así: “(...) ¿cuál es el sentido que hoy tiene la ciencia como vocación? La respuesta más simple es la que Tolstoi ha dado con las siguientes palabras: 'La ciencia carece de sentido puesto que no tiene respuesta para las únicas cuestiones que nos importan, las de qué debemos hacer y cómo debemos vivir'. Difícilmente podría discutirse el hecho de que, efectivamente, no responde a estas cuestiones. (...) tal vez, a falta de respuesta (...), contribuye, en cambio, a plantear adecuadamente estas cuestiones". Max Weber, El político y el cientifico, introducción de R. Aron (Madrid: Alianza Editorial, 1975), pos. 2150. Sobre el mismo tópico, ver Eduardo Weisz, "Las raíces históricas del destino moderno en la perspectiva trágica weberiana". VI Jornadas de Sociología de la UNLP, 9 y 10 de diciembre de 2010, en La Plata, Argentina, 2010. Disponible en: http://www.memoria.fahce.unlp.edu.ar/trab_eventos/ ev.5060/ev.5060.pdf. 
órdenes de vida y esferas de valor. ${ }^{26}$ Como escribe en un famoso párrafo: "Las relaciones inconmovibles y mohosas del pasado, con todo su séquito de ideas y creencias viejas y venerables, se derrumban y las nuevas envejecen antes de echar raíces. Todo lo que se creía permanente y perenne se esfuma, lo santo es profanado $y$, al fin, el hombre se ve constreñido, por la fuerza de las cosas, a contemplar con mirada fría su vida y sus relaciones con los demás". ${ }^{27}$

Pregunta: ¿acaso tras las denuncias del lucro, del rechazo a las relaciones egoístas, de la crítica a la mercantilización de la educación, y del repudio al intento de "economizar" la cultura y la vida entera por parte de las minorías activas estudiantiles no hay una reacción desesperada frente a un mundo que parece moverse cada día más por intereses, intercambios, contratos y circulación de mercancías en el teatro del dinero? ¿No es cierto acaso que la mercantilización generalizada de la existencia constituye hoy un problema que moviliza múltiples reacciones contrarias, por la sensación de que banaliza la existencia, reduciéndola a valores puramente prácticos, lejos de toda ética fraterna y todo sentido trascendente?

Finalmente, tercero, el fenómeno del nihilismo, entendido como pérdida del sentido en diversos planos existenciales, desde la muerte de Dios hasta la muerte del padre, desde la disolución de las comunidades hasta la desaparición de lo que algunos llaman "grandes relatos"; o sea, visiones comprensivas de la historia que buscan explicarla no como mero azar ni sólo como fatal destino. ${ }^{28} \mathrm{El}$ "fin de la historia" que recorre la cultura de nuestra época forma parte de este fenómeno.

Un psicoanalista y ensayista italiano, Massimo Recalcati, habla en relación con esto de "la evaporación de los adultos"; los que estarían desvaneciéndose, dice él, "ante el peso de sus responsabilidades educativas". ${ }^{29} \mathrm{Y}$, en similar vena diagnóstica, sugiere que "nuestra

${ }^{26}$ Timo Jütten, "Habermas and Markets", en Constellations 20, n. ${ }^{\circ} 4$ (2013): 587-603. Asimismo, Timo Jütten, "The Colonization Thesis: Habermas on Reification", en International Journal of Philosophical Studies 19, n. ${ }^{\circ} 5$ (2011): 701-727.

${ }^{27}$ Karl Marx y Friedrich Engels, Manifiesto comunista (@e-artnow: 2015), versión electrónica, pos. 275.

${ }^{28}$ Michael A. Gillespie, "Nihilism after Nietzsche", Bollettino Filosofico 30 (2015): 80-100.

${ }^{29}$ Massimo Recalcati, El complejo de Telémaco. Padres e hijos tras el ocaso del progenitor (Barcelona: Anagrama, 2014), versión digital, pos. 668. 
época es la época de la crisis simbólica de la función de la autoridad paterna". Más allá de cualquier explicación técnica, esto significa un corte o discontinuidad en la transmisión de sentidos. Una verdadera ruptura generacional, con múltiples causas y manifestaciones, pero que, al final, debe entenderse como un vacío de sentido. Significa que la generación adulta no tiene un legado que transmitir. Ha llegado a un punto cero de su cultura. Tampoco domina las nuevas tecnologías del conocimiento. Sus ideologías parecen agotadas. Su autoridad está en cuestión. Sus realidades familiares son disonantes. Su testimonio ha perdido legitimidad.

Pregunta: ¿acaso todo esto pasa desapercibido para los jóvenes? Vuelvo a citar al autor italiano: "Si el lugar de los adultos queda vacío, abandonado, repudiado, será difícil para las nuevas generaciones sentirse reconocidas, será difícil que puedan sentirse realmente como hijos. Hijos, ¿de quién? ¿De qué padres, de qué adultos? ¿De qué clase de testimonio de vida?" ${ }^{30}$ ¿No parece evidente — cada vez más, con cada año que pasa del nuevo milenio- que la herencia cultural entre generaciones se ha transformado en un legado altamente ambiguo y contradictorio, en el que junto con los beneficios de la técnica se heredan también los riesgos manufacturados por nuestra civilización; junto con el poder transformador, los escombros medioambientales; junto con los medios de autoridad, su falta de legitimidad, y así por delante?

Tal vez estas preguntas ayuden a entender que los jóvenes - particularmente sus minorías más sensibles a los legados de su época- no reciben hoy una tierra prometida e, incluso, que algunos puedan sentir que heredan una "tierra baldía"; un mundo técnicamente más sofisticado, tal vez, pero cuyo sentido parece esfumarse en el aire.

De modo que si los jóvenes se declaran conformes, en general, con los bienes materiales y simbólicos que les ofrece su sociedad, no debe olvidarse que esa percepción se conjuga con variados rasgos negativos en relación con desigualdades, abusos y percepción de que colectivamente las cosas no funcionan como debieran. Pero, más decisivo, los grados de conformismo que puedan existir a nivel de mayoría coexisten con una profusa diversidad de minorías activas que - en distintas áreas- actúan movidas por sentimientos, valores, expectativas y motivos divergentes, dando lugar a dinámicas de disonancia, rechazo y rebeldía que, como gérmenes de cambio, se mueven en todas direcciones

${ }^{30}$ Ibídem, pos. 857. 
dentro del cuerpo de la normalidad y conformidad. Por último, los jóvenes actuales viven su vida en una época cuya cultura se halla puesta en tensión por contradicciones profundas en los planos de una excesiva racionalización instrumental, un mercantilismo sin límites y una herencia intergeneracional que no transmite autoridad ni testimonio. Que nadie diga, pues, que la juventud es la edad más bella de la vida; quizá sea la más difícil y la más compleja de entender.

\section{BIBLIOGRAFÍA}

Aguilera Ruiz, Óscar. "Los estudios sobre juventud en Chile: coordenadas para un estado del arte". Última Década 17, n. ${ }^{\circ} 31$ (2009): 109-127. https://dx.doi. org/10.4067/S0718-22362009000200007

Arendt, Hannah. "Reflections on Little Rock". Dissent 6 (1959): 45-56.

Buckingham, David. "Selling Youth: The Paradoxical Empowerment of the Young Consumer". En Youth Cultures in the Age of Global Media Basingstoke, de D. Buckingham, S. Bragg \& M.J. Kehily, cap. 20. Basingstoke: Palgrave Macmillan, 2014.

Cabalin, Cristián. "Estudiantes conectados y movilizados: El uso de Facebook en las protestas estudiantiles en Chile". Comunicar, Revista Cientifica de Educomunicación 43, n. ${ }^{\circ}$ XXII (2014): 25-33.

Canales, Manuel, Antonino Opazo \& Juan Pablo Camps. "Salir del cuarto. Expectativas juveniles en el Chile de hoy". Última Década 44 (2016): 73-108.

Centro de Estudios en Juventud. "III Estudio sobre valores en jóvenes - 2013". Santiago: Universidad Católica Silva Henríquez.

Centro de Estudios Públicos. Encuesta CEP abril-mayo de 2017.

Cohen, Stanley. Folk Devils and Moral Panics. Londres: MacGibon and Kee, 1972.

Earl, Jennifer, Thomas V. Maher \& Thomas Elliot. "Youth, Activism, and Social Movements". Sociology Compass 11, n. ${ }^{\circ} 4$ (2017). doi: 10.1111/soc4.12465

Gillespie, Michael A. "Nihilism after Nietzsche". Bollettino Filosofico 30 (2015): 80-100.

González, Ricardo, coordinador. ¿Malestar en Chile? Informe encuesta CEP 2016. Santiago: Centro de Estudios Públicos, 2017.

Hodkinson, Paul. "Beyond Spectacular Specifics in the Study of Youth (sub) Cultures". Journal of Youth Studies 15, n. ${ }^{\circ} 5$ (2012): 557-72. - "Spectacular Youth? Young People's Fashion and Style". En Handbook of Youth and Young Adulthood, editado por A. Furlong. Londres: Routledge, 2009.

"Youth Cultures and the Rest of Life: Subcultures, Post-Subcultures and Beyond". Journal of Youth Studies 19, n. ${ }^{\circ} 5$ (2016): 629-645. 
Huidobro, Vicente. "Balance patriótico". Acción 4, 8 de agosto de 1925. http:// www.memoriachilena.cl/602/w3-article-317954.html.

Injuv. VIII Encuesta Nacional de Jóvenes. 2015.

Jütten, Timo. "Habermas and Markets". Constellations 20, n. 4 (2013): 587-603.

" "The Colonization Thesis: Habermas on Reification". International Journal of Philosophical Studies 19, n. 5 (2011): 701-727.

Krause, Mariane, Pablo Torche, Elda Velásquez \& Andrea Jaramillo. "Social Representations of Violence among Young Chileans Involved in Violence". Psicoperspectivas, Individuo y Sociedad 13, n. ${ }^{\circ} 2$ (2014): 55-66.

Levine, John M. \& R. Scott Tindale. "Social Influence in Groups". En APA Handbook of Personality and Social Psychology: Vol. 2, editado por M. Mikulincer \& P. R. Shaver. Group Processes, 2015: 3-34.

Marx, Karl \& Friedrich Engels. Manifiesto comunista. Clásicos de la Literatura. (a)e-artnow, 2015. Versión electrónica.

Medel Sierralta, Rodrigo Miguel \& Nicolás Manuel Somma González. “¿Marchas, ocupaciones o barricadas? Explorando los determinantes de las tácticas de la protesta en Chile". Política y Gobierno 23, n. ${ }^{\circ} 1$ (2016): 163-199.

Moscovici, Serge. Social Influence and Social Change. London: Academic Press, 1976.

Nizan, Paul. Aden, Arabie [1931]. Ebooks libres et gratuits, 2016. https://www. ebooksgratuits.com/pdf/nizan_aden_arabie.pdf

OECD. Education at a Glance 2016. Organisation for Economic Co-operation and Development: París, 2016.

Recalcati, Massimo. El complejo de Telémaco. Padres e hijos tras el ocaso del progenitor. Barcelona: Anagrama, 2014.

Sandoval Manríquez, Mario. "Jóvenes y valores: Reflexiones sociológicas a partir de datos empíricos". En Juventudes: metáforas del Chile contemporáneo, editado por Pablo Cottet. Santiago: Proyecto Juventudes - RIL Editores, 2015: 253-278.

Sartre, Jean-Paul "Préface”. En Aden, Arabie, de Paul Nizan. París: Maspéro, 1976.

Scaff, Lawrence A. "Max Weber's Legacy". Presentación en la reunión anual de la Kantoh Sociological Society. Tokio, junio 22 de 2014. https://www.jstage.jst. go.jp/article/kantoh/2015/28/2015_18/_pdf

Schröter, Susanne. "Rituals of Rebellion - Rebellion as Rituals: A theory reconsidered". En The Dynamics of Changing Rituals, editado por J. Kreinath, C. Hartung \& A. Deschner. Nueva York: Peter Lang, 2004.

Weber, Max. El político y el científico (con introducción de R. Aron). Madrid: Alianza Editorial, 1975.

_ La ética protestante y el espiritu del capitalismo (introducción y edición crítica de Francisco Gil Villegas). México D.F: Fondo de Cultura Económica, 2012. 
Weisz, Eduardo. "Las raíces históricas del destino moderno en la perspectiva trágica weberiana". VI Jornadas de Sociología de la UNLP. La Plata, Argentina, 9 y 10 de diciembre de 2010. http://www.memoria.fahce.unlp.edu. ar/trab_eventos/ev.5060/ev.5060.pdf. EP 